Pacific Journal of Mathematic 


\title{
THE SPLITTING OF OPERATOR ALGEBRAS, II
}

\author{
STEVE WRIGHT
}

Let $\left\{A_{\alpha}: \alpha \in A\right\}$ be a family of $C^{*}$-algebras (resp., $W^{*}$. algebras). For $\alpha_{0} \in A$, we let $P_{\alpha_{0}}: \bigoplus_{\alpha} A_{\alpha} \rightarrow A_{\alpha_{0}}$ denote the canonical coordinate projection of $\oplus_{\alpha} A_{\alpha}$ onto $A_{\alpha_{0}}$. If $B$ is a $C^{*}$-(resp., $W^{\left.*_{-}\right)}$subalgebra of $\oplus_{\alpha} A_{\alpha}$, we say that $B$ splits if $B=\bigoplus_{\alpha} P_{\alpha}(B)$. In this note, we give conditions both necessary and sufficient for $B$ to split. In the $C^{*}$-category, these conditions are given in terms of separation properties of the spectrum and primitive ideal space of $B$, and in the $W^{*}$-category, the conditions are expressed in terms of disjointness of certain subsets of the center of $B$. We also give examples to show that these conditions cannot be weakened, and are hence the best possible of their kind.

In [4], Sze-kai Tsui and the author obtained several results on the splitting of singly-generated operator algebras. Theorems 2.1 and 3.4 of [4] are the principle results of that paper, and it is the purpose of this paper to present results which both improve and generalize the main results of [4].

If $A$ is a $C^{*}$-algebra (resp., $W^{*}$-algebra) and $a \in A$, then $C^{*}(a)$ (resp., $\left.W^{*}(a)\right)$ denotes the $C^{*}$-subalgebra (resp., $W^{*}$-subalgebra) of $A$ generated by $a$. Let $\pi$ be a representation of $A_{\alpha_{0}}$, for some fixed $\alpha_{0} \in A$. We define a representation $\tilde{\pi}$ of $\bigoplus_{\alpha} A_{\alpha}$ by

$$
\tilde{\pi}: \bigoplus_{\alpha} a_{\alpha} \longrightarrow \pi\left(a_{\alpha_{0}}\right), \quad \bigoplus_{\alpha} a_{\alpha} \in \bigoplus_{\alpha} A_{\alpha} \text {. }
$$

The sets

$$
\sum_{n_{0}}=\left\{\operatorname{ker}\left(\left.\tilde{\rho}\right|_{C^{*}\left(\oplus_{\alpha} a_{\alpha}\right)}\right): \rho \text { an irreducible representation of } C^{*}\left(a_{\alpha_{0}}\right)\right\}
$$

are subsets of the primitive ideal space of $C^{*}\left(\bigoplus_{\alpha} a_{\alpha}\right)$. The first main result of [4] asserted that $C^{*}\left(a_{1} \oplus a_{2}\right)$ splits if and only if $\Sigma_{1}$ and $\Sigma_{2}$ disconnect the pimitive ideal space of $C^{*}\left(a_{1} \oplus a_{2}\right)$ equipped with the hull-kernel topology. In Theorem 2.2 of this paper, we improve and generalize this to arbitrary $C^{*}$-subalgebras of arbitrary direct sums of $C^{*}$-algebras.

Let $N$ be $W^{*}$-algebra with predual $N_{*}$ and let $\tau$ be a $\sigma\left(N, N_{*}\right)$ continuous representation of $N$. We set $\operatorname{supp} \tau=$ complement of the central support projection of ker $\tau$ in $N$. We denote the class of all nonzero $\sigma\left(N, N_{*}\right)$-continuous representations of $N$ by $\operatorname{Rep}_{\sigma}(N)$. If $S$ and $T$ are subsets of $N$, we say that $S$ and $T$ are orthogonal if $s t=t s=0$, for $s \in S$ and $t \in T$.

Let $N_{\alpha}: \alpha \in \mathscr{A}$ be a family of $W^{*}$-algebras, with $\bigoplus_{\alpha} n_{\alpha}$ a fixed 
element of $\bigoplus_{\alpha} N_{\alpha}$. We set

$$
S_{\alpha_{0}}=\left\{\operatorname{supp}\left(\left.\widetilde{\tau}\right|_{W^{*}\left(\oplus_{\alpha^{n}}{ }^{\prime}\right)}\right): \tau \in \operatorname{Rep}_{\sigma}\left(W^{*}\left(n_{\alpha_{0}}\right)\right)\right\} \text {. }
$$

The second main theorem of [4] asserted that $W^{*}\left(n_{1} \oplus n_{2}\right)$ splits if and only if $S_{1}$ and $S_{2}$ are orthogonal and $\sup \left(S_{1} \cup S_{2}\right)=$ identity in $W^{*}\left(n_{1}\right) \oplus W^{*}\left(n_{2}\right)$. In Theorem 2.4 of this paper we improve and generalize this to arbitrary $W^{*}$-subalgebras of arbitrary direct sums of $W^{*}$-algebras.

2. Solution of the splitting problem. Let $\left\{A_{\alpha}: \alpha \in \mathfrak{R}\right\}$ be a family of $C^{*}$-algebras, and let $P_{\alpha}: \bigoplus_{\alpha} A_{\alpha} \rightarrow A_{\alpha}$ denote the canonical coordinate projection of $\bigoplus_{\alpha} A_{\alpha}$ onto $A_{\alpha}$. A $C^{*}$-subalgebra $B$ of $\bigoplus_{\alpha} A_{\alpha}$ is said to be substantial in $\bigoplus_{\alpha} A_{\alpha}$ if $P_{\alpha}(B)=A_{\alpha}$, for each $\alpha \in \mathfrak{A}$. A $C^{*}$-subalgebra $B$ of $\bigoplus_{\alpha} A_{\alpha}$ is said to split if $B=\bigoplus_{\alpha} P_{\alpha}(B)$. The question that concerns us asks: when does a $C^{*}$-subalgebra of $\bigoplus_{\alpha} A_{\alpha}$ split?

The following lemma, the key to our answer to this question, is a trivial modification of a result kindly suggested to us by Don Hadwin, who in turn heard it from T. B. Hoover:

LeMma 2.1. Let $\left\{A_{1}, \cdots, A_{n}\right\}$ be $C^{*}$-algebras, with $B$ a substantial $C^{*}$-subalgebra of $A_{1} \oplus \cdots \oplus A_{n}$. Then $B=A_{1} \oplus \cdots \oplus A_{n}$ if and only if the following condition holds: there exist no distinct indices $i$ and $j$ and irreducible representations $\rho_{\alpha}$ of $A_{\alpha}, \alpha=i, j$, for which $\left.\tilde{\rho}_{i}\right|_{B}=\left.\tilde{\rho}_{j}\right|_{B}$.

Proof. $(\Rightarrow)$. This is clear.

$(\Leftarrow)$. Fix $i \neq j$. It sufficies to show that $\left(P_{i} \oplus P_{j}\right)(B)=$ $A_{i} \oplus A_{j}$, and hence we may assume with no loss of generality that $n=2$. Set $J_{i}=B \cap \operatorname{ker}\left(P_{i}\right), i=1,2$. Then $J_{1}+J_{2}$ is a closed, two-sided ideal in $B$. Let $a_{1} \in A_{1}$. Since $P_{1}(B)=A_{1}$, there exists $a^{\prime} \in A_{2}$ such that $a_{1} \oplus a^{\prime} \in B$. Define the $*$-homomorphism $\sigma_{1}: A_{1} \rightarrow$ $B /\left(J_{1}+J_{2}\right)$ by $\sigma_{1}: a_{1} \rightarrow a_{1} \oplus a^{\prime}+\left(J_{1}+J_{2}\right)$. Let $a_{2} \in A_{2}$. Since $P_{2}(B)=A_{2}$, there exists $a^{\prime \prime} \in A_{1}$ such that $a^{\prime \prime} \oplus a_{2} \in B$. Define the *-homomorphism $\sigma_{2}: A_{2} \rightarrow B /\left(J_{1}+J_{2}\right)$ by $\sigma_{2}: a_{2} \rightarrow a^{\prime \prime} \oplus a_{2}+\left(J_{1}+J_{2}\right)$. One easily checks that $\left.\tilde{\sigma}_{1}\right|_{B}=\left.\tilde{\sigma}_{2}\right|_{B}$. Suppose $\tilde{\sigma}_{1}(B) \neq(0)$. Let $\rho$ be an irreducible representation of $\widetilde{\sigma}_{1}(B)$. Since $\sigma_{1}\left(A_{1}\right)=\widetilde{\sigma}_{1}(B)=\widetilde{\sigma}_{2}(B)=$ $\sigma_{2}\left(A_{2}\right), \rho_{i}=\rho \circ \sigma_{i}$ is an irreducible representation of $A_{i}, i=1,2$, and we thus have $\left.\tilde{\rho}_{1}\right|_{B}=\left.\tilde{\rho}_{2}\right|_{B}$, contrary to assumption. Thus $\tilde{\sigma}_{1}(B)=$ $\tilde{\sigma}_{2}(B)=(0)$, whence $\sigma_{1}=\sigma_{2}=0$. It follows that $J_{1}=(0) \oplus A_{2}$, $J_{2}=A_{1} \oplus(0)$, whence $B=A_{1} \oplus A_{2}$.

We now introduce some notation and terminology for the state- 
ment and proof of our principle result.

Let $A$ be a $C^{*}$-algebra. We let $A^{* *}$ denote the enveloping $W^{*}$ algebra of $A$, realized as the ultraweak closure of the image of $A$ under its universal representation. If $S$ is a subset of $A^{* *}$, we will denote the ultraweak closure of $S$ by $S^{-}$. If $I$ is a closed, twosided ideal in $A$ then $I^{-}$is an ultraweakly closed, two-sided ideal in $A^{* *}$, so there is a central projection $p$ of $A^{* *}$ such that $I^{-}=A^{* *} p$. We set $s(I)=p$.

If $p$ is a central projection of $A^{* *}$, the representation of $A$ defined by $a \rightarrow a p, a \in A$, will be denoted by $\pi_{p}$.

If $B$ is a $C^{*}$-subalgebra of $A$, we will write $B / B \cap I=A / I$ to indicate that the canonical injection of $B / B \cap I$ into $A / I$ is surjective.

The class of all irreducible representations of $A$ will be denoted by $\operatorname{Irr}(A)$, and we identify $\operatorname{Irr}(A / I)$ with $\{\rho \in \operatorname{Irr}(A): I \leqq \operatorname{ker}(\rho)\}$.

We recall that two representations of $A$ are disjoint if they have no nonzero, unitarily equivalent subrepresentations.

Finally, we need to consider the restricted direct sum $\hat{\bigoplus}_{\alpha} A_{\alpha}$ of a family $A_{\alpha}: \alpha \in \mathfrak{A}$ of $C^{*}$-algebras. By definition, $\hat{\bigoplus}_{\alpha} A_{\alpha}$ is the closed, two-sided ideal of $\bigoplus_{\alpha} A_{\alpha}$ consisting of all elements $\bigoplus_{\alpha} a_{\alpha}$ for which the sets $\left\{\alpha \in \mathfrak{X}:\left\|a_{\alpha}\right\| \geqq \varepsilon\right\}$ are finite for each $\varepsilon>0$.

We can now present our solution of the splitting problem for arbitrary families of $C^{*}$-algebras:

THEOREM 2.2. Let $A_{\alpha}: \alpha \in \mathfrak{A}$ be a family of $C^{*}$-algebras, $B$ a $C^{*}$-subalgebra of $\bigoplus_{\alpha} A_{\alpha}$. Let $A=\bigoplus_{\alpha} P_{\alpha}(B), \quad I=\hat{\bigoplus}_{\alpha} P_{\alpha}(B)$. The following are equivalent:

(i) $B$ splits;

(ii) $B / B \cap I=A / I$, and the sets

$\left\{\operatorname{ker}\left(\left.\rho\right|_{B}\right): \rho \in \operatorname{Irr}(A / I)\right\}, \quad\left\{\operatorname{ker}\left(\left.\tilde{\rho}\right|_{B}\right): \rho \in \operatorname{Irr}\left(P_{\alpha}(B)\right)\right\}, \quad \alpha \in \mathfrak{A}$,

are pairwise disjoint subsets of the primitive ideal space of $B$;

(iii) $B / B \cap I=A / I$, and the following condition holds: for each fixed $\alpha \in \mathfrak{A}$ and $\left(\alpha_{1}, \alpha_{2}\right) \in \mathfrak{A} \times \mathfrak{A}$ with $\alpha_{1} \neq \alpha_{2}$, and for each ordered pair $\left(\rho_{1}, \rho_{2}\right)$ in $\operatorname{Irr}\left(P_{\alpha_{1}}(B)\right) \times \operatorname{Irr}\left(P_{\alpha_{2}}(B)\right)$ (resp., $\left.\operatorname{Irr}(A / I) \times \operatorname{Irr}\left(P_{\alpha}(B)\right)\right)$, we have $\left.\tilde{\rho}_{1}\right|_{B} \neq\left.\tilde{\rho}_{2}\right|_{B}$ (resp., $\left.\left.\rho_{1}\right|_{B} \neq\left.\tilde{\rho}_{2}\right|_{B}\right)$.

Proof. The implications (i) $\Rightarrow$ (ii) and (ii) $\Rightarrow$ (iii) are clear.

(iii) $\Rightarrow$ (i). We may assume with no loss of generality that $B$ is substantial in $A=\bigoplus_{\alpha} A_{\alpha}$. Let $p=s(I)$, so that $I^{-}=A^{* *} p$. The map $a+I \rightarrow a(1-p)$ of $A / I$ into $A^{* *}(1-p)$ extends to an isomorphism of $(A / I)^{* *}$ onto $A^{* *}(1-p)$. Since $B / B \cap I=A / I$, we conclude that $B^{-}(1-p)=A^{* *}(1-p)$.

Let $\Sigma$ denote the set of all finite subsets of the indexing set 
2. For each $\sigma \in \Sigma$ and $a=\bigoplus_{\alpha} a_{\alpha} \in \bigoplus_{\alpha} A_{\alpha}$, set $a_{o}=\bigoplus\left\{a_{\alpha}: \alpha \in \sigma\right\}$, $A_{\sigma}=\bigoplus\left\{A_{\alpha}: \alpha \in \sigma\right\}, \quad P_{\sigma}=\bigoplus\left\{P_{\alpha}: \alpha \in \sigma\right\}$, and $B_{\sigma}=P_{\sigma}(B)$. It follows from the hypothesis that $B_{\sigma}$ is a substantial $C^{*}$-subalgebra of $A_{\sigma}$ which satisfies the conditions of Lemma 2.1, so by that lemma, $B_{\sigma}=A_{\sigma}$. Thus $\left.P_{o}\right|_{B}$ implements a $*$-isomorphism of $B / \operatorname{ker}\left(\left.P_{o}\right|_{B}\right)$ onto $A_{o}$, and since this isomorphism is an isometry, it follows that $B$ has the following property:

(*) for each $a=\bigoplus_{\alpha} a_{\alpha} \in \bigoplus_{\alpha} A_{\alpha}$ and $\sigma \in \Sigma$, there exists

$$
\begin{aligned}
& b_{\sigma}=\bigoplus_{\alpha} b_{\alpha}^{\sigma} \in B \text { such that }\left\|b_{\sigma}\right\| \leqq 1+\|a\| \text { and } \\
& b_{\alpha}^{\sigma}=a_{\alpha}, \text { for each } \alpha \in \sigma .
\end{aligned}
$$

Set $P_{\alpha}=$ support projection of $A_{\alpha}^{* *}$ in $A^{* *}$. Then $\left\{p_{\alpha}: \alpha \in \mathfrak{X}\right\}$ is a family of pairwise orthogonal projections of $I^{-}$such that $\bigoplus_{\alpha} p_{\alpha}=p$. Letting $p_{\sigma}=\bigoplus\left\{p_{\alpha}: \alpha \in \sigma\right\}$ for each $\sigma \in \Sigma$, and considering $\Sigma$ as a net, ordered by inclusion, we have $\lim _{\sigma}\left\|x-x p_{\sigma}\right\|=0$, for each $x \in I$.

Fix $x \in I$. By $\left({ }^{*}\right)$, for each $\sigma \in \Sigma$ there exists $b_{\sigma} \in B$ such that $x p_{\sigma}=b_{o} p_{\sigma}$ and $\left\|b_{o}\right\| \leqq 1+\|x\|$. Since $\left\{b \in B^{-}:\|b\| \leqq 1+\|x\|\right\}$ is ultraweakly compact, $\left\{b_{o}\right\}$ has an ultraweak accumulation point $b \in B^{-}$. Passing if necessary to a cofinal subnet, we may assume that ultraweak- $\lim _{\sigma} b_{o}=b$, and we hence have

$$
x=\lim _{\sigma} x p_{\sigma}=\lim _{\sigma} b_{\sigma} p_{\sigma}=\text { ultraweak-lim } b_{\sigma} p_{\sigma}=b p .
$$

Thus $I \subseteq B^{-} p$, whence $A^{* *} p=I^{-}=B^{-} p$.

We assert that $\left.\pi_{p}\right|_{B}$ and $\left.\pi_{1-p}\right|_{B}$ are disjoint. If they are not, we can find irreducible representations $\rho_{1}$ and $\rho_{2}$ of $A$ with $I \nsubseteq \operatorname{ker}\left(\rho_{1}\right)$, $I \subseteq \operatorname{ker}\left(\rho_{2}\right)$, such that $\left.\rho_{1}\right|_{B}=\left.\rho_{2}\right|_{B}$. Since $\rho_{1}=\tilde{\rho}$ for $\rho \in \operatorname{Irr}\left(A_{\alpha}\right)$ for some $\alpha \in \mathfrak{A}$, this contradicts (iii).

Let $q=$ support projection of $B^{-}$in $A^{* *}$. Since $A^{* *}=B^{-} p \oplus$ $B^{-}(1-p), q=1$, and so $1 \in B^{-}$. Thus by the disjointness of $\left.\pi_{p}\right|_{B}$ and $\left.\pi_{1-p}\right|_{B}$ and Proposition 5.2.1 of [1], we have (with ' denoting the commutant):

$$
\begin{aligned}
B^{-} & =\left(\pi_{p} \oplus \pi_{1-p}\right)(B)^{\prime \prime}=(B p)^{\prime \prime} \oplus(B(1-p))^{\prime \prime} \\
& =B^{-} p \oplus B^{-}(1-p) \\
& =A^{* *} .
\end{aligned}
$$

If $\iota: B \rightarrow A$ denotes the inclusion map, then $B^{-}$can be identified with $\iota^{* *}\left(B^{* *}\right)$ in $A^{* *}$. We have hence shown that $\iota^{* *}$ is a surjection of $B^{* *}$ onto $A^{* *}$. By duality and the Hahn-Banach theorem, we therefore conclude that $B=A$.

If instead of the full direct sum $\bigoplus_{\alpha} A_{\alpha}$, we consider $C^{*}$-subalgebras of restricted direct sums $\hat{\oplus}_{\alpha} A_{\alpha}$, then $I^{* *}=A^{* *}$ in the above proof, and so we immediately deduce: 
Corollary 2.3. Let $A_{\alpha}: \alpha \in \mathfrak{A}$ be a family of $C^{*}$-algebras, $B$ a $C^{*}$-subalgebra of $\hat{\oplus}_{\alpha} A_{\alpha}$. The following are equivalent:

(i) $B$ splits;

(ii) The sets $\left\{\operatorname{ker}\left(\left.\tilde{\rho}\right|_{B}\right): \rho \in \operatorname{Irr}\left(P_{\alpha}(B)\right)\right\}, \quad \alpha \in \mathfrak{A}$, are pairwise disjoint subsets of the primitive ideal space of $B$;

(iii) For each $\left(\alpha_{1}, \alpha_{2}\right) \in \mathfrak{A} \times \mathfrak{A}$ with $\alpha_{1} \neq \alpha_{2}$ and for each $\left(\rho_{1}, \rho_{2}\right) \in$ $\operatorname{Irr}\left(P_{\alpha_{1}}(B)\right) \times \operatorname{Irr}\left(P_{\alpha_{2}}(B)\right)$, we have $\left.\tilde{\rho}_{1}\right|_{B} \neq\left.\tilde{\rho}_{2}\right|_{B}$.

The reasoning of Theorem 2.2 can be applied to easily obtain a solution to the splitting problem for an arbitrary direct sum of $W^{*}$-algebras. Indeed, recalling the notation of the introduction, we have:

TheOREM 2.4. Let $N_{\alpha}: \alpha \in \mathfrak{A}$ be a family of $W^{*}$-algebras, $M$ a $W^{*}$-subalgebra of $\oplus_{\alpha} N_{\alpha}$. The following are equivalent:

(i) $M$ splits;

(ii) The subsets $\left\{\operatorname{supp}\left(\left.\widetilde{\tau}\right|_{M}\right): \tau \in \operatorname{Rep}_{o}\left(P_{\alpha}(M)\right)\right\}, \alpha \in \mathscr{A}$, of the center of $M$ are pairwise disjoint;

(iii) For each $\left(\alpha_{1}, \alpha_{2}\right) \in \mathfrak{A} \times \mathfrak{A}$ with $\alpha_{1} \neq \alpha_{2}$ and for each $\left(\tau_{1}, \tau_{2}\right) \in$ $\operatorname{Rep}_{\sigma}\left(P_{\alpha_{1}}(M)\right) \times \operatorname{Rep}_{\sigma}\left(P_{\alpha_{2}}(M)\right)$, we have $\left.\tilde{\tau}_{1}\right|_{M} \neq\left.\widetilde{\tau}_{2}\right|_{M}$.

Proof. The implications (i) $\Rightarrow$ (ii) and (ii) $\Rightarrow$ (iii) are clear.

(iii) $\Rightarrow$ (i). Lemma 2.1 holds with $C^{*}$-(sub)algebra (resp., irreducible representation) replaced by $W^{*}$-(sub)algebra (resp., nonzero, $\sigma\left(A_{\alpha},\left(A_{\alpha}\right)_{*}\right)$-continuous representation). Thus the argument of the first part of the implication (iii) $\Rightarrow$ (i) of Theorem 2.2, appropriately modified, together with the fact that the net $\left\{p_{\sigma}: \sigma \in \Sigma\right\}$ (where $p_{\alpha}=$ identity of $N_{\alpha}$ ) converges in the ${ }^{*}$-strong topology to the identity of $\bigoplus_{\alpha} N_{\alpha}$ now finishes the proof.

RemaRks. (1) The splitting phenomenon is much more likely to occur in the $W^{*}$-category than in the $C^{*}$-category, to which Theorems 2.2 and 2.4 attest. In fact, an example of two diagonal operators $T_{1}$ and $T_{2}$ acting on a separable Hilbert space is given in [4] for which $W^{*}\left(T_{1} \oplus T_{2}\right)$ splits, while neither $W^{*}\left(\operatorname{Re} T_{1} \oplus \operatorname{Re} T_{2}\right)$, $W^{*}\left(\operatorname{Im} T_{1} \oplus \operatorname{Im} T_{2}\right)$, nor $C^{*}\left(T_{1} \oplus T_{2}\right)$ splits.

(2) Theorems 1.4 and 2.2 of [3] can be combined with Lemma 2.1 to give an alternate proof of Theorem 2.2. The proof given here seems more natural in the present context, quickly gives a solution to the splitting problem for $W^{*}$-algebras, and avoids the fairly complicated machinery of algebras of operator fields and regularized dual spaces used in [3].

(3) In closing, we present some simple examples which show 
that the conditions of Theorem 2.2 cannot be weakened. More specifically, we give examples of a proper, substantial $C^{*}$-subalgebra $B$ of $l_{\infty}$ for which $B / B \cap c_{0}=l_{\infty} / c_{0}$ and for which

$$
\left(P_{1} \oplus \cdots \oplus P_{n}\right)(B)=C^{n} \text {, for each positive integer } n,
$$

and a proper, substantial $C^{*}$-subalgebra $C$ of $l_{\infty}$ which satisfies the second part of condition (ii) of Theorem 2.2 and for which $C / C \cap c_{0}$ has codimension 1 in $l_{\infty} / c_{0}$.

We identify $l_{\infty}$ with the $C^{*}$-algebra $C(X)$ of continuous, complexvalued functions on the Stone-Čech compactification $X$ of the positive integers $Z_{+}$with discrete topology. $Z_{+}$is a discrete, dense, open subset of $X$. Set $E=X \backslash Z_{+}$. Then $c_{0}$ can be identified with the ideal of functions in $C(X)$ which vanish on $E$.

Choose $x \in Z_{+}, y \in E$, and set $B=\{f \in C(X): f(x)=f(y)\} . \quad B$ is a proper $C^{*}$-subalgebra of $C(X)$. Let $\left\{x_{1}, \cdots, x_{n}\right\}$ be a fixed finite subset of $Z_{+},\left(a_{1}, \cdots, a_{n}\right)$ a fixed $n$-tuple of complex numbers. Then by the Tietze extension theorem ([2], Theorem 5.1, p. 149), we can find an $f \in C(X)$ such that $f\left(x_{i}\right)=a_{i}, i=1, \cdots, n$, and $f(x)=f(y)$. Thus $B$ is substantial in $C(X)$ and satisfies $\left({ }^{* *}\right)$. Let $g$ be a fixed element of $C(X)$. Again by the Tietze extension theorem, there exists $f \in C(X)$ such that $f=g$ on $E$ and $f(x)=g(y)$. Thus $f \in B$, and since $f-g=0$ on $E, f-g \in c_{0}$. Hence $B / B \cap c_{0}=l_{\infty} / c_{0}$.

To obtain $C$, simply choose distinct elements $x$ and $y$ of $E$ and set $C=\{f \in C(X): f(x)=f(y)\}$. Since elements of $\operatorname{Irr}\left(l_{\infty}\right)$ of the form $\tilde{\rho}, \rho$ an irreducible representation of some coordinate algebra, correspond to evaluation at points of $Z_{+}$and elements of $\operatorname{Irr}\left(l_{\infty} / c_{0}\right)$ correspond to evaluation at points of $E$, the previous reasoning shows that $C$ satisfies the second part of condition (ii) of Theorem 2.2. Now $l_{\infty} / c_{0}$ can be identified with the $C^{*}$-algebra $C(E)$ of continuous, complex-valued functions on $E$, and $C / C \cap c_{0}$ can be identified with the subalgebra $D$ of all $f \in C(E)$ for which $f(x)=f(y)$. Since $D$ is the kernel of the linear functional $f \rightarrow f(x)-f(y)$ on $C(E)$, it follows that $C / C \cap c_{0}$ has codimension 1 in $l_{\infty} / c_{0}$.

These arguments can clearly be used to construct similar examples for an arbitrary infinite direct sum of commutative $C^{*}$ algebras.

\section{References}

1. J. Dixmier, C*-algebras, North-Holland, Amsterdam-New York-Oxford, 1977.

2. J. Dugundji, Topology, Allen and Bacon, Boston, 1966.

3. J. M. G. Fell, The structure of algebras of operator fields, Acta Math., 106 (1961), 233-280.

4. S.-K. Tsui and S. Wright, The splitting of operator algebras, Pacific J. Math., 84 (1979), 201-215. 
Received June 12, 1980 and in revised form June 24, 1981. Partially supported by an Oakland University Faculty Research Fellowship.

OARLAND UNIVERSITY

ROCHESTER, MI 48063 



\section{PACIFIC JOURNAL OF MATHEMATICS}

\section{EDITORS}

DONALD BABBITT (Managing Editor)

University of California

Los Angeles, California 90024

Hugo RossI

University of Utah

Salt Lake City, UT 84112

C. C. Moore and Arthur Agus

University of California

Berkeley, CA 94720
J. DugundJI

Department of Mathematics University of Southern California Los Angeles, California 90007

R. FInN and J. Milgram Stanford University Stanford, California 94305

ASSOCIATE EDITORS
R. ARNES
E. F. BeCKenbach
B. H. NeumanN
F. WOLF
K. YoSHIDA

\section{SUPPORTING INSTITUTIONS}

UNIVERSITY OF ARIZONA

UNIVERSITY OF BRITISH COLUMBIA

CALIFORNIA INSTITUTE OF TECHNOLOGY

UNIVERSITY OF CALIFORNIA

MONTANA STATE UNIVERSITY

UNIVERSITY OF NEVADA, RENO

NEW MEXICO STATE UNIVERSITY

OREGON STATE UNIVERSITY
UNIVERSITY OF OREGON

UNIVERSITY OF SOUTHERN CALIFORNIA

STANFORD UNIVERSITY

UNIVERSITY OF HAWAII

UNIVERSITY OF TOKYO

UNIVERSITY OF UTAH

WASHINGTON STATE UNIVERSITY

UNIVERSITY OF WASHINGTON 


\section{Pacific Journal of Mathematics}

\section{Vol. 103, No. $1 \quad$ March, 1982}

Abdul Aziz, On the zeros of composite polynomials ..................

Salomon Benzaquen and Enrique M. Cabaña, The expected measure of the level sets of a regular stationary Gaussian process $\ldots \ldots \ldots \ldots \ldots$

Claudio D’Antoni, Roberto Longo and László Zsidó, A spectral mapping theorem for locally compact groups of operators $\ldots \ldots \ldots \ldots \ldots \ldots \ldots 17$

Ronald Dotzel, Semifree finite group actions on homotopy spheres ........ 25

Daniel H. Gottlieb, The Lefschetz number and Borsuk-Ulam theorems . . . . . 29

Shui-Hung Hou, On property $(Q)$ and other semicontinuity properties of

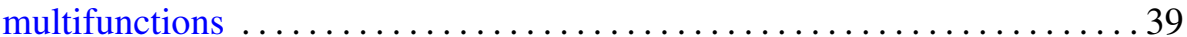

Kevin Mor McCrimmon, Compatible Peirce decompositions of Jordan triple systems

Mitsuru Nakai, Corona problem for Riemann surfaces of Parreau-Widom

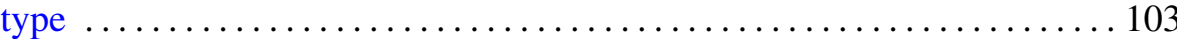

Jack Ray Porter and R. Grant Woods, Extensions of Hausdorff spaces . . . . 111

Milton Rosenberg, Quasi-isometric dilations of operator-valued measures

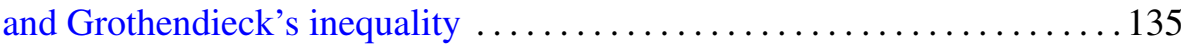

Joseph L. Taylor, A bigger Brauer group $\ldots \ldots \ldots \ldots \ldots \ldots \ldots \ldots \ldots \ldots$

Thomas Vogel, Symmetric unbounded liquid bridges . . . . . . . . . . . 205

Steve Wright, The splitting of operator algebras. II ............... 243 